\title{
Flow Transition to Turbulence and induced Acoustics at Mach 6
}

\author{
Dimitris Drikakis, ${ }^{1, \text { a) }}$ Konstantinos Ritos, ${ }^{2,}$ b) S. Michael Spottswood, ${ }^{3}$ and Zachary B. Riley ${ }^{4}$ \\ 1) University of Nicosia, Nicosia CY-2417, Cyprus \\ 2) Department of Mechanical \& Aerospace Engineering, University of Strathclyde, Glasgow, G1 1XJ, UK \\ ${ }^{3)}$ Air Force Research Laboratory, Wright Patterson AFB, Ohio 45433-7402, USA \\ 4) ARCTOS Technology Solutions, LLC., Dayton, OH, 45432, USA
}

(Dated: 16 August 2021)

This paper presents the results of implicit Large Eddy Simulation (iLES) and Direct Numerical Simulation (DNS) for flow and acoustics for transitional and turbulent boundary layer over a flat plate at Mach 6. The DNS was about 50 times more refined grid-wise than iLES. Both DNS and iLES were performed using the same numerical schemes, initial and boundary conditions. We compare the different numerical approaches concerning the shape factor, momentumthickness-based Reynolds number, heat flux on the wall, Reynolds stress, and near-wall acoustics. We perform pressure fluctuations spectral analysis and propose a predictive model. We show that iLES captures pretty accurately the flow and acoustic characteristics in the turbulent region. Differences up to $5 \mathrm{~dB}$ occur between iLES and DNS in the transition region. iLES also shifts slightly further downstream the end of the transition and underpredicts the shear stress value peak. The iLES captures the near-wall acoustic spectrum roll-off accurately at low and medium frequencies. It underpredicts high frequencies' content due to grid constraints. Overall, iLES gives excellent results compared to the significantly more refined DNS. The results show that high-order numerical simulations can help adapt and validate semi-empirical models for the engineering design and acoustic loading on hypersonic structures.

\section{INTRODUCTION}

Structural elements of supersonic and hypersonic aircraft are exposed to acoustic fatigue due to pressure fluctuations beneath the transitional or turbulent boundary layers (TBLs). There is literature concerning subsonic and supersonic TBLs, including studies regarding the amplitude of pressure fluctuations ${ }^{1}$ and the roll-off of the pressure spectrum $^{2-4}$. These models and theories have been validated experimentally ${ }^{5-8}$, as well as verified numerically ${ }^{9,10}$.

Although hypersonic TBLs have been studied experimentally during the recent years ${ }^{1-14}$, numerical studies are limited $^{15-17}$. Furthermore, high-resolution investigations of TBLs on acoustic loading are indeed scarce ${ }^{18}$. To the best of our knowledge, the frequency content of pressure fluctuations in transitional boundary layers at supersonic and hypersonic speeds was discussed for the first time in ${ }^{19}$.

Zhao et al. ${ }^{20}$ published wind tunnel experiments for the wall pressure fluctuations for hypersonic flow over a flat plate at Mach numbers 6 and 8. They used a linear sensor array and processed the data by a beam-forming algorithm. They showed that the transition causes a significant increase in the equivalent sound pressure level (SPL) for the wall pressure fluctuations, which reaches a peak value at the end of the transition region and further dissipates in the turbulent area.

In another recent publication, Huang et al. ${ }^{21}$ used DNS to characterize the pressure fluctuations under the turbulent portion of the boundary layer over a sharp 7 degrees half-angle cone at a nominal freestream Mach number of 7.91. The DNS was performed using the non-uniform digital-filtering method to generate inflow turbulence with the mean boundary layer

\footnotetext{
${ }^{a}$ Electronic mail: drikakis.d@unic.ac.cy. Also affiliated with the University of Nicosia Research Foundation and Defence and Security Research Institute. ${ }^{b)}$ Electronic mail: konstantinos.ritos@strath.ac.uk
}

profiles and the Reynolds stress tensor at the inflow provided by the pre-cursor RANS. Huang et al. ${ }^{21}$ compared the characteristics of turbulent pressure fluctuations at the cone surface with those over a Mach 8 flat plate and showed that the inner scaling collapses the wall-pressure power spectra density (PSD) among the different flow configurations.

The linear stability theory ${ }^{22}$ shows that multiple instability modes exist in high-speed boundary layers, contrary to low-speed or incompressible boundary layers where only one mode exist (Tollmien-Schlichting). Moreover, the transition process in hypersonic boundary layers is highly random due to higher modes' existence. The random nature of the hypersonic transition process explains its sensitivity to changes in the disturbance environment that can significantly change the transition process ${ }^{17}$. Contrary to previous studies that focused on single-mode or 'controlled' transition, our research focuses on multi-mode perturbations, which comprise many waves imitating the von Kármán atmospheric turbulence.

The objectives of our study are:

- Examine the accuracy of 9th-order iLES against DNS that is about 50 times finer using the same computational model, i.e., the same numerical scheme, initial and boundary conditions. The initial conditions are based on a von Kármán atmospheric spectrum at the inlet. To our knowledge, such comparisons between iLES and DNS are performed for the first time.

- Investigate the physics of flow and acoustics using a range of parameters.

- Provide the community with data for verification and validation of hypersonic simulations. Furthermore, we show how the data can help develop reduced-order models to predict the acoustic spectrum. We present the effectiveness and accuracy of such a model and a detailed spectral analysis of the pressure fluctuations. 


\section{COMPUTATIONAL METHODOLOGY}

We solve the compressible Navier-Stokes equations (NSE) for an ideal gas using the finite volume method (FVM). In integral form, the NSE are formulated as:

$$
\begin{gathered}
\frac{\partial}{\partial t} \iiint_{V} \rho \mathrm{dV}=-\oiint_{A} \rho(\mathbf{u} \cdot \hat{\mathbf{n}}) \mathrm{dA} \\
\frac{\partial}{\partial t} \iiint_{V} \rho \mathbf{u} \mathrm{dV}=-\oiint_{A} \rho \mathbf{u}(\mathbf{u} \cdot \hat{\mathbf{n}}) \mathrm{dA}-\oiint_{A} p \hat{\mathbf{n}} \mathrm{dA}+\oiint_{A}(\tau \cdot \hat{\mathbf{n}}) \mathrm{dA}
\end{gathered}
$$

$$
\begin{aligned}
\frac{\partial}{\partial t} \iiint_{V} \rho e \mathrm{dV}=- & \oiint_{A} \rho(\mathbf{u} \cdot \hat{\mathbf{n}}) e \mathrm{dA}-\oiint_{A} p(\mathbf{u} \cdot \hat{\mathbf{n}}) \mathrm{dA} \\
& +\oiint_{A}(\mathbf{u} \cdot \tau) \cdot \hat{\mathbf{n}} \mathrm{dA}-\oiint_{A}(\mathbf{q} \cdot \hat{\mathbf{n}}) \mathrm{dA}
\end{aligned}
$$

where $\rho$ is the density; $\mathbf{u}$ is the velocity vector; $p$ is the static pressure; $\hat{\mathbf{n}}$ is the outward pointing unit normal of surface element $\mathrm{dA}$ of the closed finite control volume $\mathrm{dV} ; e$ is the total energy per unit mass given by $e=i+\mathbf{u} \cdot \mathbf{u} / 2 ; i$ is the specific internal energy, which for a calorically perfect gas is given by:

$$
i=c_{v} T=\frac{p}{\rho(\gamma-1)}
$$

$T$ is the temperature, $c_{v}$ is the specific heat capacity at constant volume, and $\gamma$ is the heat capacity ratio (or adiabatic index) defined as $\gamma=c_{p} / c_{v}$ where $c_{p}$ is the specific heat capacity at constant pressure and $R_{s}=c_{p}-c_{v}=c_{v}(\gamma-1)$ is the specific gas constant. At high Mach numbers and temperatures, vibrational excitation and dissociation effects of diatomic molecules result in ionization and make the calorically perfect gas assumption no longer valid. In the above case, the ratio of specific heats will depend on the temperature. The above effects are not taken into consideration here as the maximum temperature is well below the critical temperature for oxygen dissociation $(2500 \mathrm{~K})$. Furthermore, it is common to study transitional and turbulent boundary layers at Mach greater than 4 using the calorically perfect gas model, particularly in the range of Mach 4 to $6^{18,21,23-27}$. This facilitates studying turbulent near-wall effects in isolation from other assumptions associated with ionized gas models. Furthermore, distinguishing a particular hypersonic flow from supersonic cannot be based purely on chemical non-equilibrium processes (e.g., oxygen dissociation). The dominant flow transition mechanism changes near Mach 4 from the oblique 1st mode to the 2 nd mode instability. Additionally, surface heating affects these instabilities differently ${ }^{28}$.

For a Newtonian fluid, the shear stress tensor is given by:

$$
\tau=\lambda_{b}(\nabla \cdot \mathbf{u}) I+\mu\left[\nabla \mathbf{u}+(\nabla \mathbf{u})^{T}\right]
$$

where $I$ is the identity tensor; the bulk viscosity is given by $\lambda_{b}=-4 \mu / 3$ according to Stokes hypothesis; and $\mu$ is the dynamic viscosity obtained by Sutherland's Law as:

$$
\mu(T)=\mu_{r e f}\left(\frac{T}{T_{r e f}}\right)^{\frac{3}{2}} \frac{T_{r e f}+T_{s}}{T+T_{s}}
$$

where the free-stream values are used as the reference and the Sutherland temperature is $T_{s}=110.4 \mathrm{~K}$.

The heat flux is calculated by Fourier's Law of heat conduction:

$$
\mathbf{q}=-\kappa \nabla T
$$

where $\kappa$ is the heat conductivity given by:

$$
\kappa(T)=\frac{c_{p}}{\operatorname{Pr}} \mu(T)
$$

and $\operatorname{Pr}=0.72$ is Prandtl's number.

We have employed DNS and iLES in the framework of the CFD code CNS3D ${ }^{29-32}$. In classical Large Eddy Simulations (LES), the smallest length scales, which are the most computationally expensive to resolve, are removed via low-pass filtering of the Navier-Stokes equations. Then, the unresolved scales of turbulence are modeled by subgrid-scale models. In iLES, the computational grid removes small scales. The modelling of the unresolved scales is obtained through the non-linear dissipation embedded onto the high-resolution and high-order numerical schemes used to discretize the convective terms. There is a significant body of work in the literature, both theoretical and numerical, explaining iLES methods and demonstrating their accuracy in turbulent flows, e.g., see the books $^{33,34}$

A detailed description of the method used in this study can be found in the recent paper by Kokkinakis et al. ${ }^{29}$. CNS3D solves the full Navier-Stokes equations using a finite volume Godunov-type method for the convective terms. We discretize the inter-cell numerical fluxes by solving the Riemann problem using the reconstructed values of the primitive variables at the cell interfaces. We use a one-dimensional swept unidirectional stencil for the reconstruction of the variables. The Riemann problem is solved using the so-called "Harten, Lax, van Leer, and (the missing) Contact" (HLLC) approximate Riemann solver ${ }^{35,36}$.

The high-order numerical discretization is based on a 9th-order Weighted-Essentially-Non-Oscillatory (WENO) scheme, and the specific numerical details are explained by Kokkinakkis et al. ${ }^{29}$. We briefly mention below the WENO characteristics in the framework of CNS3D. ${ }^{29}$ The left and right reconstruction stencil are normalized, per variable, according to a transformation function ${ }^{29}$. The transformation normalizes the candidate stencils so that the entire stencil's maximum value becomes equal to one. The minimum value takes a positive and nonzero value, and the value range scales relative to the maximum. The normalization of the total stencil values per variable prevents negative WENO smoothness indicator values, reduces the numerical dissipation and simplifies applying the proceeding step. Furthermore, Kokkinakis's 
et al. WENO version uses a total variation (TV) limiting procedure for each candidate stencil and obtain the maximum TV ratio between the candidate stencils. If all of the stencils contain significant discontinuities, then the maximum TV ratio can be incorrectly small. Thus, an additional criterion is introduced through the linear WENO weights ${ }^{29}$, i.e., the standard WENO weights are also modified according to the mapped WENO (WENO-M) approach ${ }^{37}$.

Furthermore, CNS3D uses a second-order central scheme to discretize the viscous terms. The solution is advanced in time using a five-stage (fourth-order accurate) optimal strongstability-preserving Runge-Kutta method ${ }^{38-40}$ and references therein. The 9th order discretization scheme applies to convective terms. In contrast, the viscous terms and temporal discretization is of 2 nd and 4 th order, respectively. Therefore, the overall order of accuracy is probably second order. However, despite the above, several studies have shown that the order of accuracy and the numerical design of the method used for the discretization of the convective (non-linear) terms significantly influence the accuracy of the simulations ${ }^{30-33,41-43}$. The above is due to the non-linearity of these terms responsible for capturing transitional and turbulent flow features, as well as shock waves and contact discontinuities. CNS3D has been extensively validated against experimental and numerical results $29-32,44$.

\section{HIGH-RESOLUTION SIMULATIONS AT MACH 6}

\section{A. Flow analysis}

The problem case concerns hypersonic flow over a flat plate at Mach 6 subjected to von Kármán atmospheric spectrum at the inlet. We compare iLES and DNS results in the same CFD code (CNS3D) using the same simulation parameters, with the only difference being the mesh resolution. This comparison allows examining the accuracy of high-fidelity approaches for predicting the complexity of transition, turbulence and associated acoustic effects. The turbulence intensity of the freestream velocity at the inlet is $T u=1 \%$. Regarding acoustic loading, it has been shown that the inlet's turbulent intensity affects the maximum acoustic load location, but not the actual value ${ }^{19}$. A comprehensive study (incompressible flow) on free stream turbulence and TBL can be found in ${ }^{45}$. Based on the freestream properties (Table I) and the reference length $x_{l}=2 \mathrm{~mm}$, the incoming flow has a Reynolds number of $R e_{x_{l}}=77,791$. The reference length is calculated from the leading edge of the plate. We implement periodic boundary conditions in the spanwise direction $(z)$. In the wall-normal direction $(y)$, we use a no-slip isothermal wall (with a temperature $\mathrm{T}_{w}$ of $1600 \mathrm{~K}$, near the adiabatic value $)^{46}$. High-order implementation of the boundary conditions requires fictitious cells inside the wall. The velocity components on these cells are linearly extrapolated from the computational cells inside the domain. The temperature is also linearly extrapolated using the specified wall temperature. The density is calculated from the equation of state considering a zero pressure gradient normal to the wall. We apply supersonic outflow condi- tions at the outlet and far-field boundaries. The same methodology was also recently used in conjunction with the digital filter approach to study the flow and acoustics of supersonic shock/boundary-layer interactions ${ }^{29}$.

We visualize the start and the end of the different flow regions using the instantaneous density gradient along the simulation domain's centerline (Fig. 1). The transition from laminar to turbulent flow is captured. The flow can be considered fully turbulent in the last third of the simulation domain; see discussion below for the instantaneous two-point spatial autocorrelation functions (ACF) and shape factor. In this paper, the end-of-the-transition region is defined as the location of maximum skin friction. The thickening of the boundary layer is significant along the simulation domain. This is also highlighted in Figure 2 where we show the contour plot of the streamwise velocity component in the turbulent region. The continuous black line has been drawn based on $99 \%$ of the freestream velocity and approximately indicates the edge of the boundary layer.

To check that the limited length of the simulated flat plate does not affect the dynamics of the observed vortex structures, we calculate the instantaneous two-point spatial autocorrelation functions (ACF) for the velocity field:

$$
A C F(x)=\overline{\left(\frac{\left\langle u_{i}^{\prime}\left(x_{0}\right) u_{i}^{\prime}(x)\right\rangle}{\left\langle u_{i}^{\prime}\left(x_{0}\right)^{2}\right\rangle}\right)},
$$

where $u_{i}^{\prime}$ can be any of the fluctuating velocity components $\left(u^{\prime} ; v^{\prime} ; w^{\prime}\right)$. For $x=x_{0}$, the ACF is trivially unity, and it decays with increasing distance from $x_{0}$. The autocorrelation function converges to zero for the limit of considerable distances since turbulence is a random phenomenon. The above is demonstrated in Figure 3, where the autocorrelation function converges to zero after a very short distance from the initial point. The initial point has been chosen at $x=30$, just after the skin friction value peak, indicating the transition region's end. The autocorrelation function is averaged in the spanwise direction. This behavior justifies that the numerical predictions from the present simulations can be used to investigate turbulence structure both near and far from the wall ${ }^{47}$. The velocity field behaviour changes in the various sub-layers of the boundary layer, and we get anticorrelation near the wall $\left(y^{+}<20\right)$ and inside either the viscous sublayer or the buffer layer. While the ACF vanish much quicker, as expected, in the outer layer and outside the boundary layer. The above ACF oscillations observed normal to the wall in the transition region might also be due to other several reasons, such as numerical effects or indicating instabilities that tend to dump out in the turbulent region. The above points require further investigation, notably the potential transition instabilities. The above was beyond the scope of the present study.

To examine mesh resolution effects and differences between iLES and DNS simulations on these type of flows, we compare two cases, one with $\sim 20$ million cells (iLES) and one with $\sim 1.0$ billion cells (DNS). The fine DNS mesh aims at capturing the smallest possible turbulent scales and provides a high-resolution reference for examining the accuracy of iLES in high-speed turbulent boundary layers. In general, performing DNS on complex engineering geometries would 


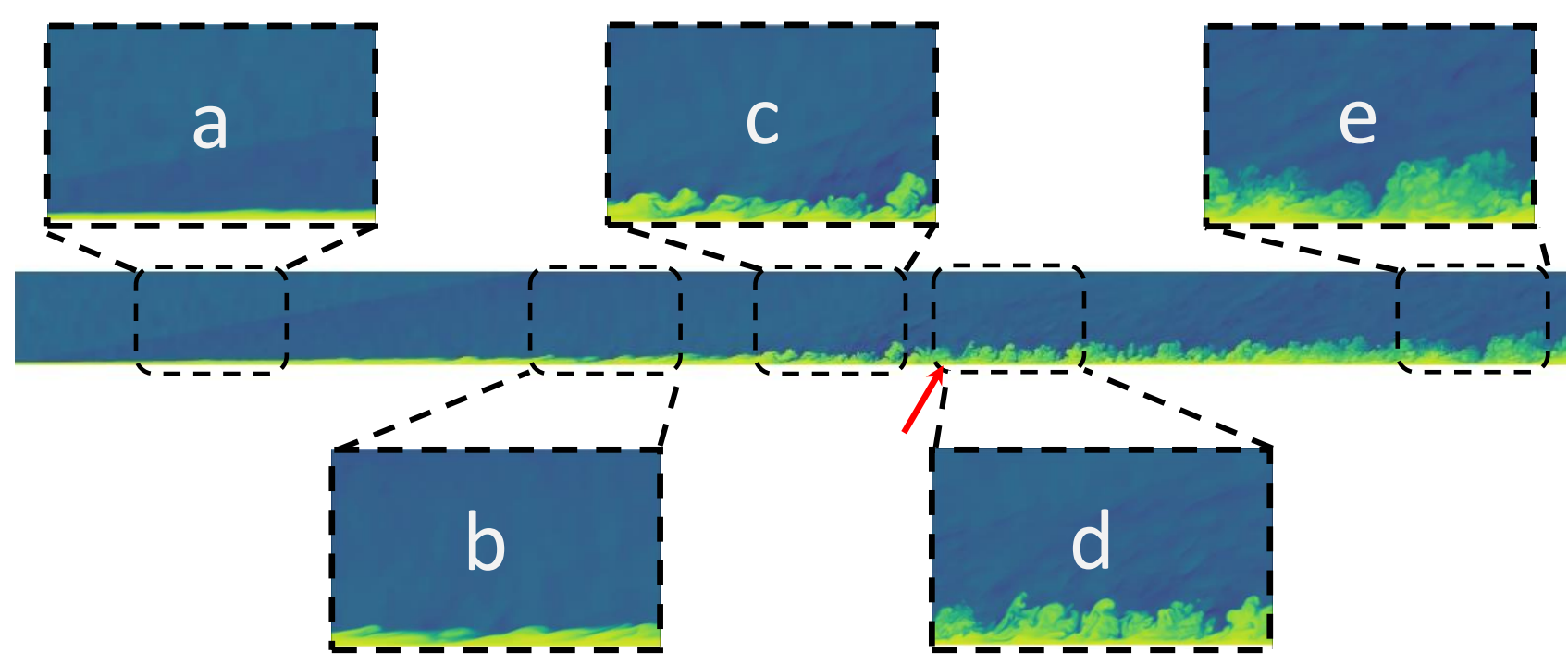

FIG. 1. Contour plot of the instantaneous density gradient along the centerline of the DNS simulation domain. Different regions have been identified as a) laminar, b) beginning of transition, c) peak of transition, d) end of transition and e) fully turbulent. The red arrow is placed at $x=30$ indicating the end-of-the-transition point.

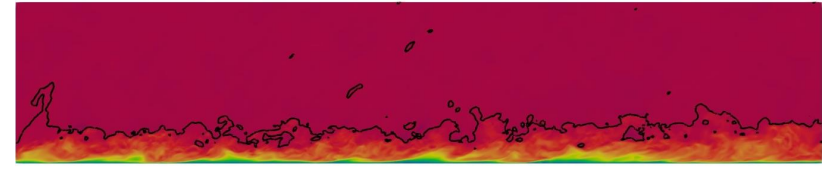

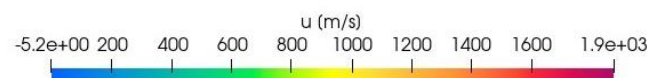

FIG. 2. Contour plot of the streamwise velocity component in the turbulent region. The black line highlights the boundary layer edge.

require hundreds of billions of mesh points. Thus, exploring the accuracy of alternative methods such as iLES compared with high-resolution DNS on simpler geometries provides the scientific and engineering communities with guidance for the best simulation practices. The DNS case's computational cost is significant, requiring access to National Supercomputers with thousands of CPU cores and special postprocessing techniques to handle the terabytes of data produced. On the other hand, we can perform iLES on a workstation with at least $20 \mathrm{CPU}$ cores. For the DNS results presented in this study we utilised 11,760 cores and $\sim 2.5$ TB of RAM on ARCHER, while for the iLES results we utilised 240 cores and $\sim 50$ GB of RAM on Archie-WeSt. Further details about both meshes, including a DNS case from the literature, are given in Table II. Both meshes are comparable, in terms of size and quality, to the DNS of fully-turbulent hypersonic flows ${ }^{18,48}$. We should also mention that both cases resolve the boundary layer with the near-wall $y^{+}$being always below 1 .

A qualitative comparison of the flow development for both iLES and DNS is shown in Figure 4 using the isosurfaces of compressible Q-criterion and contour plots velocity, density and temperature. Transition to turbulence is slightly delayed
TABLE I. Simulation parameters for hypersonic flow over a flat plate at altitude $11651 \mathrm{~m}$.

\begin{tabular}{ccccccccc}
\hline \hline $\begin{array}{c}x_{l} \\
(\mathrm{~mm})\end{array}$ & $\begin{array}{c}u_{\infty} \\
(\mathrm{m} / \mathrm{s})\end{array}$ & $\begin{array}{c}P_{\infty} \\
(\mathrm{kPa})\end{array}$ & $\begin{array}{c}T_{\infty} \\
(\mathrm{K})\end{array}$ & $\begin{array}{c}M_{\infty} \\
(-)\end{array}$ & $\begin{array}{c}\rho_{\infty} \\
\left(\mathrm{kg} / \mathrm{m}^{3}\right)\end{array}$ & $\begin{array}{c}T_{w} \\
(\mathrm{~K})\end{array}$ & $\begin{array}{c}R e_{x_{l}} \\
(-)\end{array}$ & $\begin{array}{c}\mathrm{Tu} \\
(-)\end{array}$ \\
\hline 2.0 & $1,769.92$ & 19.417 & 216.64 & 6.0 & 0.3124 & 1600 & 77,791 & $1 \%$ \\
\hline
\end{tabular}

TABLE II. Mesh parameters for hypersonic flow over a flat plate

\begin{tabular}{ccccccccc}
\hline \hline & $N_{x}$ & $N_{y}$ & $N_{z}$ & Cells (millions) & $\Delta x^{+}$ & $\Delta y_{w}^{+}$ & $\Delta y_{e}^{+}$ & $\Delta z^{+}$ \\
iLES & 811 & 201 & 121 & 19.5 & 12.6 & 0.39 & 4.7 & 5.5 \\
DNS & 4055 & 405 & 605 & 989.2 & 2.25 & 0.16 & 1.89 & 0.9 \\
DNS*18,48 & 1600 & 500 & 800 & 640 & 9.63 & 0.51 & 5.33 & 5.14 \\
\hline
\end{tabular}

in the iLES case than the reference DNS case, while a coarser grain of turbulent structures is captured in the turbulent region (Figure 4). However, considering the 50 times coarser mesh utilized by iLES, the accuracy and amount of details captured are impressive. If we focus on the temperature, density and velocity contour plots, the results are indistinguishable between the two cases.

Both cases similarly predict the shape factor $H$ (Figure 5(a)). The shape factor $H$ is used to assess the compressible boundary layer flow; $H$ is defined as the ratio of the displacement thickness $\left(\delta^{*}\right)$ to the momentum thickness $(\theta)$ :

$$
H=\frac{\delta^{*}}{\theta}=\int_{0}^{\infty}(1-\Pi) d y\left[\int_{0}^{\infty} \Pi\left(1-\frac{u(y)}{u_{\infty}}\right) d y\right]^{-1},
$$

where $\Pi=\frac{\rho(y) u(y)}{\rho_{\infty} u_{\infty}}$ while $u_{\infty}$ and $\rho_{\infty}$ are the freestream velocity and density, respectively. Previous studies ${ }^{49-52}$ have confirmed the reduction of the shape factor with increasing Reynolds number and the potential asymptotic limit at high Reynolds numbers. A correlation formula for fully turbulent 


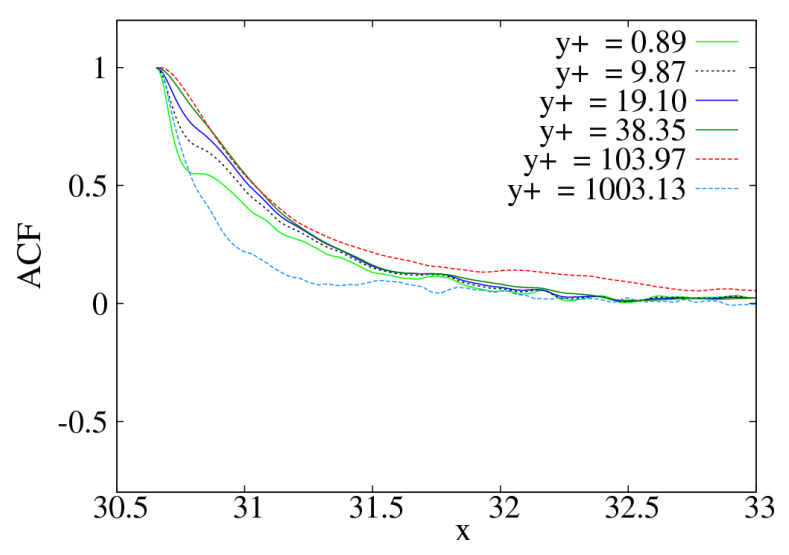

(a)

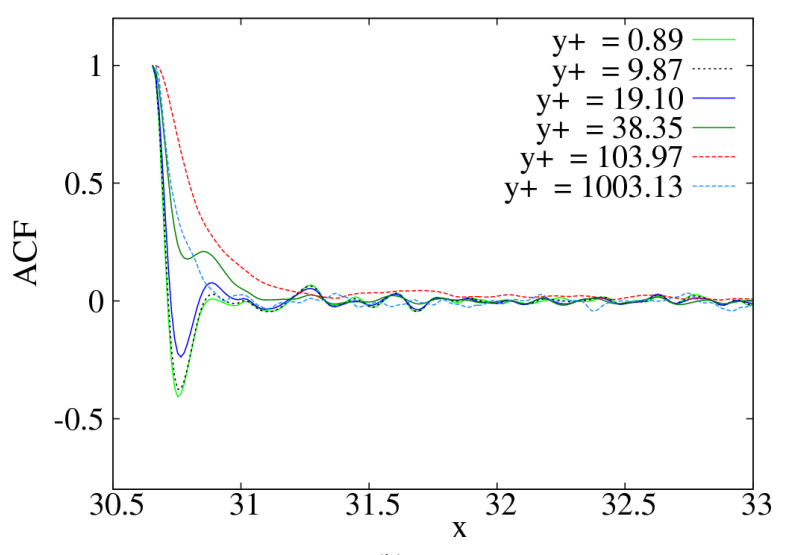

(b)

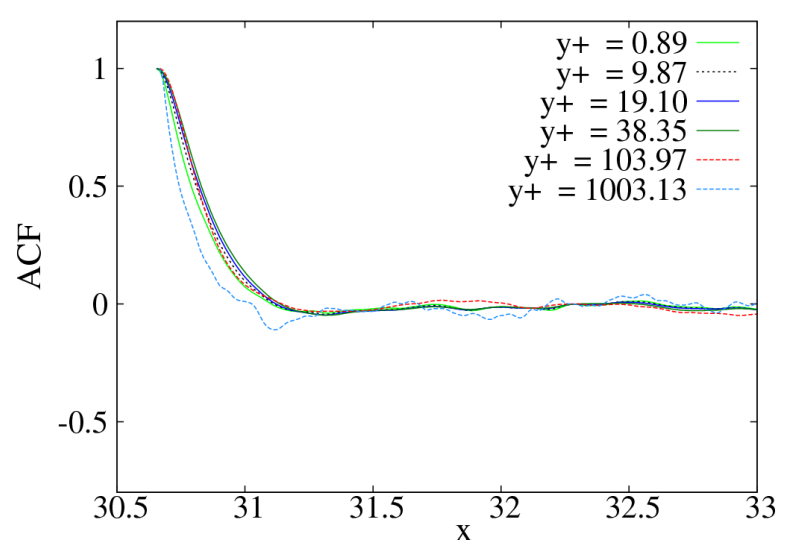

(c)

FIG. 3. Autocorrelation functions of the a) streamwise, b) normal to the wall and c) spanwise velocities at different locations normal to the wall, inside and outside the boundary layer

boundary layers and a broad range of Reynolds numbers has been proposed in the literature ${ }^{52}$

$$
H_{\text {comp }}=H_{i n c}+0.4 M^{2}+1.222 \frac{T_{w}-T_{a w}}{T_{\infty}},
$$

where $H_{\text {inc }}=1.4$. The present simulation results are in good agreement with the above formula's predictions (Figure 5(a)).
Note that flow statistics are computed by averaging in time over three flow cycles for iLES and one flow cycle for DNS, while spatial averaging in the spanwise direction is applied in both cases. The initial simulation time necessary to reach a fully developed flow (excluded from post-processing calculations) is equal to three flow cycles for both cases.

A closer observation of skin friction (Figure 5(b)) highlights that both cases capture the onset of transition at the same location. The end-of-the-transition delay is less than $5 \%$ in iLES and corresponds to 1.23 length units or $\approx 2.5 \mathrm{~mm}$. We make similar observations in Figure 5(c) for the Stanton number

$$
S t=\frac{q_{w}}{\rho_{\infty} u_{\infty} c_{p}\left(T_{a w}-T_{w}\right)},
$$

where $T_{a w}$ is the adiabatic wall temperature and peak location difference increases to 4.42 length units or $\approx 8.9 \mathrm{~mm}$. The length of the transition region, $T r$, can be estimated based on the minimum and maximum values of skin friction. Those values along with the locations of maximum pressure and thermal stresses are summarised in Table III.

TABLE III. Locations of maximum skin friction, thermal and pressure stresses. The location of minimum skin friction and the length of the transition region is also given. All streamwise locations given

\begin{tabular}{|c|c|c|c|c|c|}
\hline \multicolumn{3}{|c|}{$C_{f-\text { Start }} C_{f-E n d}$} & $T r$ & $S t_{\text {Max }}$ & $P_{r m}^{\prime}$ \\
\hline iLES & 11.88 & 29.78 & 17.90 & 28.30 & 28.80 \\
\hline DNS & 11.29 & 28.55 & 17.26 & 23.88 & 25.36 \\
\hline
\end{tabular}
in the table are non-dimensionalised with $x_{l}$.

The Stanton number produces noisy results, despite applying the same averaging approach with the previous statistics. To overcome this issue in other flow and acoustic loading statistics, we performed a Bezier smoothing operation, which allows us to identify key flow characteristics and differences between the cases more manageable. To assure the reader that the smoothing function does not affect our observations and conclusions, we include the supplementary material figures with the original unprocessed DNS data and a comparison between unprocessed data with the results after the smoothing operation. DNS and iLES give similar values for the momentum-thickness-based Reynolds number in the transition region. At the same time, iLES produces a thicker boundary layer in the turbulent region leading to an over prediction of $R e_{\theta}$ at the last third of the flow (Figure 5(d)).

We have further assessed the accuracy of iLES through higher-order statistics, starting with the calculation of the Reynolds normal and shear stresses $\tau_{i j}=\rho \overline{u_{i}^{\prime} u_{j}^{\prime}} /\left(\rho_{w} u_{\tau}^{2}\right)$, where $u_{i}^{\prime}$ can be any of the fluctuating velocity components $\left(u^{\prime}, v^{\prime}, w^{\prime}\right)$. The above is a common way to present the fluctuating velocity statistics (Figure 6), which accounts for turbulent fluctuations in fluid momentum. The iLES under-predict the magnitude of the peak Reynolds stresses associated with unresolved turbulent scales that produce the small near-wall fluctuations. However, iLES can accurately replicate DNS's shape and provide an accurate overall representation of the 


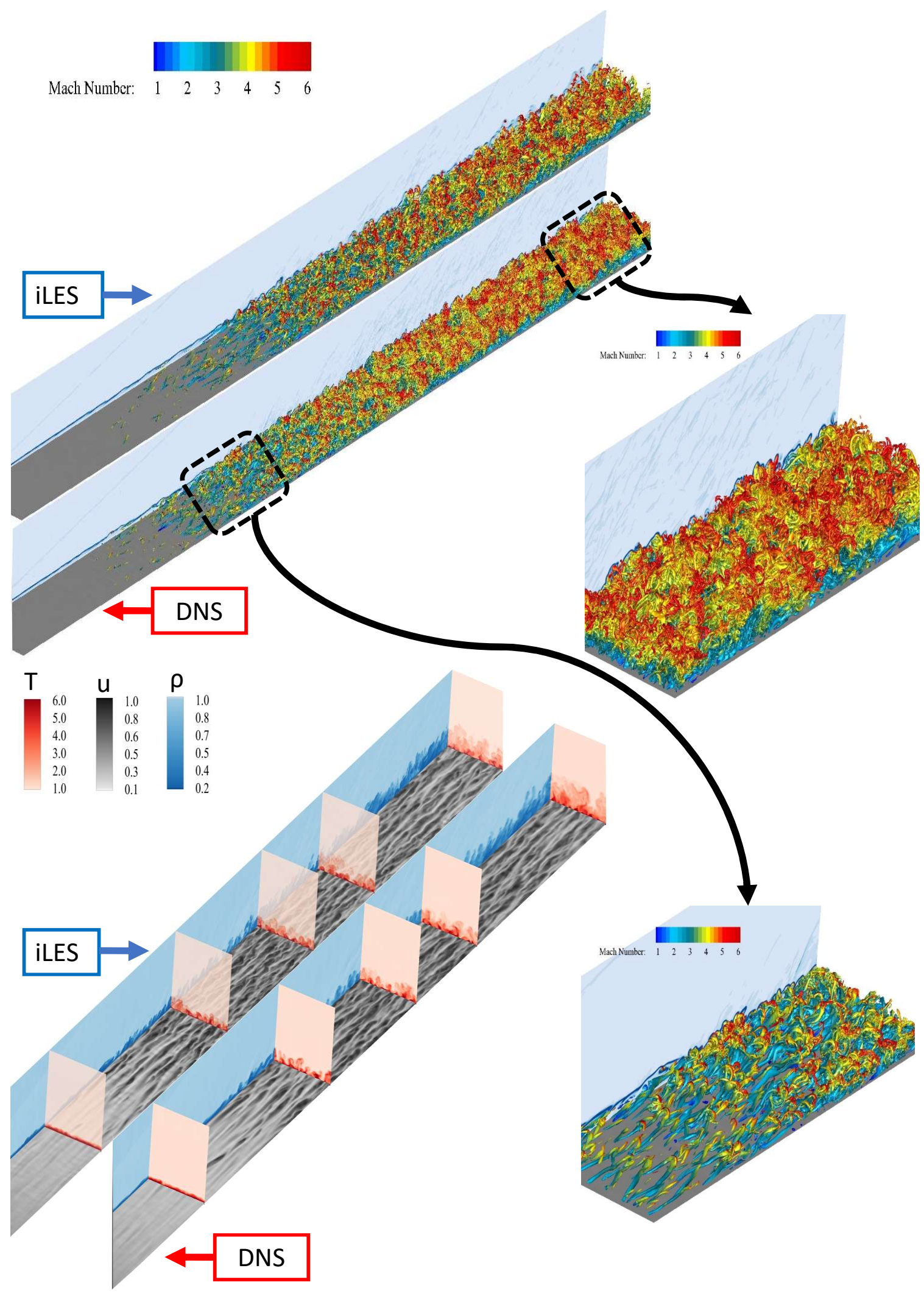

FIG. 4. Comparison of instantaneous plots between iLES and DNS. Upper figures and zoom-ins are isosurfaces of compressible Q-criterion coloured by Mach number. The density gradient is also plotted in blue scale on the graph's side, highlighting the transition region. Lower left figures are the contour plots of density and streamwise velocity near the wall and temperature contour plots at various cross-sections of the simulation domain. 


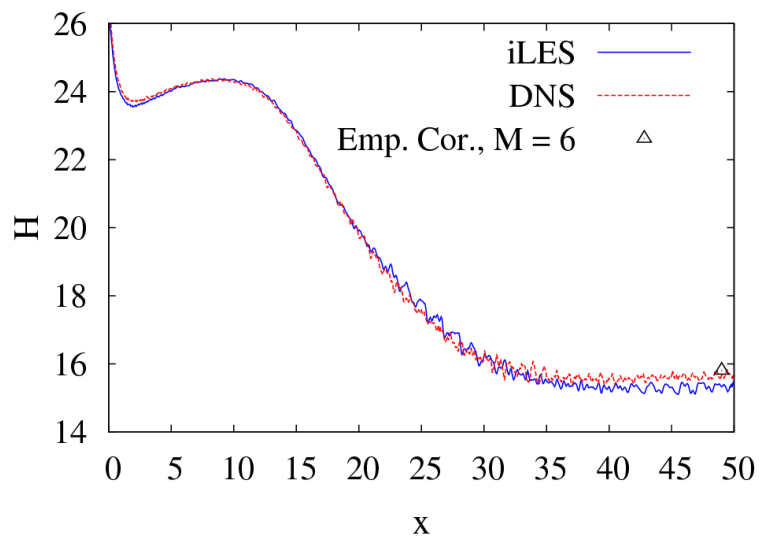

(a)

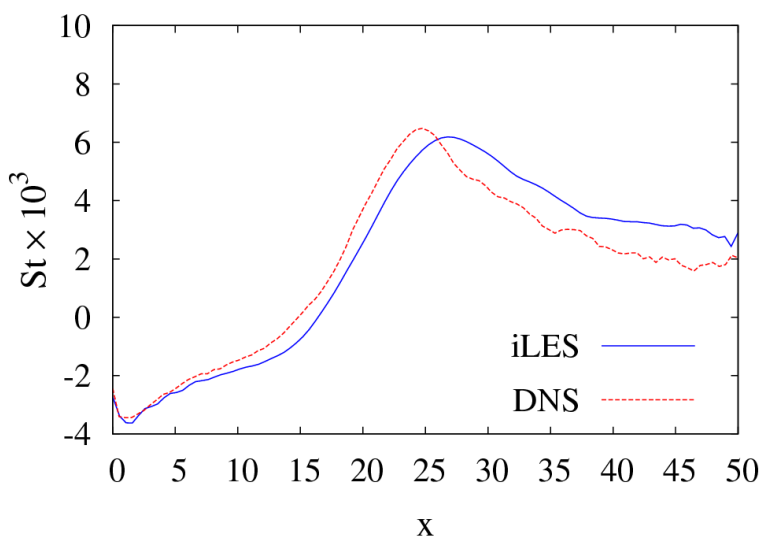

(c)

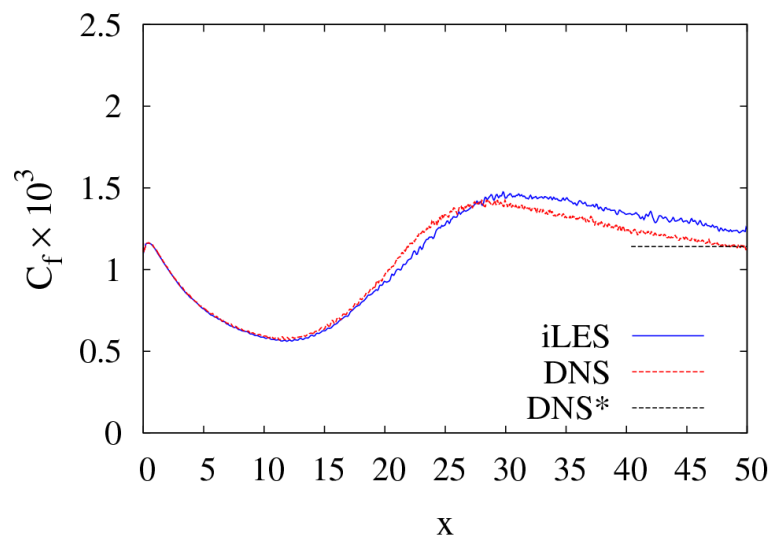

(b)

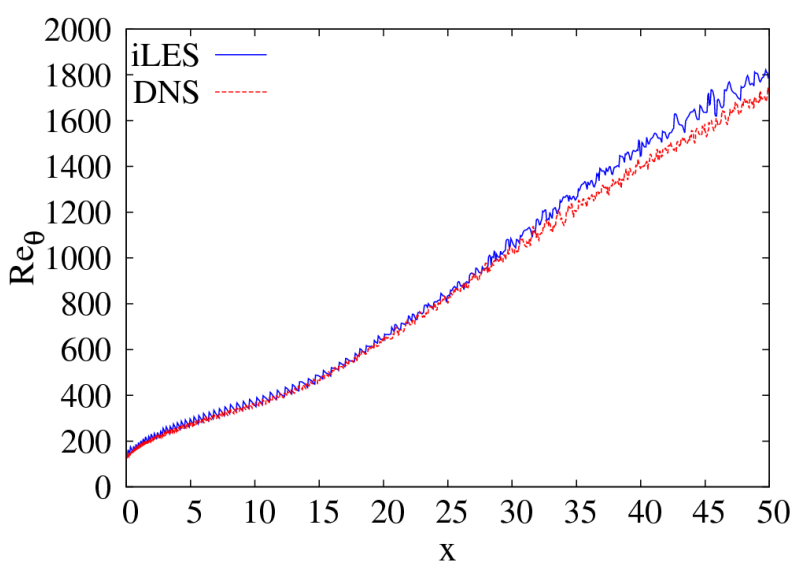

(d)

FIG. 5. Comparison between iLES ${ }^{44}$ and DNS of a) shape factor $H$, b) skin friction $C_{f}$, c) Stanton number $S t$ and d) $R e_{\theta}$ along the plate. An empirical correlation (Eq. 7) is also presented in a) for the fully turbulent flow regime. In b) an additional DNS result from a fully turbulent simulation $^{18,48}$ at $M=6$ is also included. Stanton number results in c) have been smoothed with a Bezier function, see suplementary material for additional information.

Reynolds stresses. The above is true in the viscous sublayer and the outer layer (log-law region). Figure 6 shows results from the end of the domain, but we can make similar observations in all other locations.

The physical representation of the transitional and turbulent boundary layer of the reference DNS case is further analysed by examining the skewness of the streamwise velocity fluctuations $\left(S_{u}=\overline{u^{\prime 3}} /{\overline{u^{\prime 2}}}^{1.5}\right)$ and flatness (or Kurtosis) of the normal to the wall velocity fluctuations $\left(F_{v}=\overline{v^{4}} /{\overline{v^{\prime 2}}}^{2}\right)$. The skewness or third moment is a measure of the asymmetry of the probability distribution of $u^{\prime}$, while flatness is the fourth moment that shows the frequency of events far from the mean value $^{53}$. A value of skewness around zero and a value of flatness around 3 indicate Gaussian or symmetric probability distribution function. High values of flatness indicate a highly intermittent flow. It is expected that close to the wall and in the viscous sublayer, the probability distribution of the streamwise velocity fluctuations to be positively skewed, independent of the Reynolds number of the flow ${ }^{54-56}$
The above behavior is verified in Figure 7, where the negative skewness values can quickly identify the boundary layer. As the flow develops from transitional to turbulent; the skewness becomes negative inside a large part of the boundary layer. The negative values are associated with highly negative perturbations and indicate air intrusion from the boundary layer's outer parts. Flatness is also over the Gaussian limit due to the intermittent character of turbulence in the near-wall region. Further away from the wall and in the TBL logarithmic part, turbulence returns to symmetry with $S_{u} \approx 0$ and $F_{v} \approx 3$. Close to the boundary layer edge, we observe large negative values of skewness and positive flatness values, suggesting strong intermittency in this region dominated by small amounts of $u^{\prime}$ and $v^{\prime}$ infrequent but intense positive values.

The transition region can also be identified in these plots. More specifically, $S_{u} \approx 2$ near the wall, which is also the highest value observed in the domain. We expect the above behavior since the transition to turbulence is highly intermittent by definition. We can see that the transition region affects the 


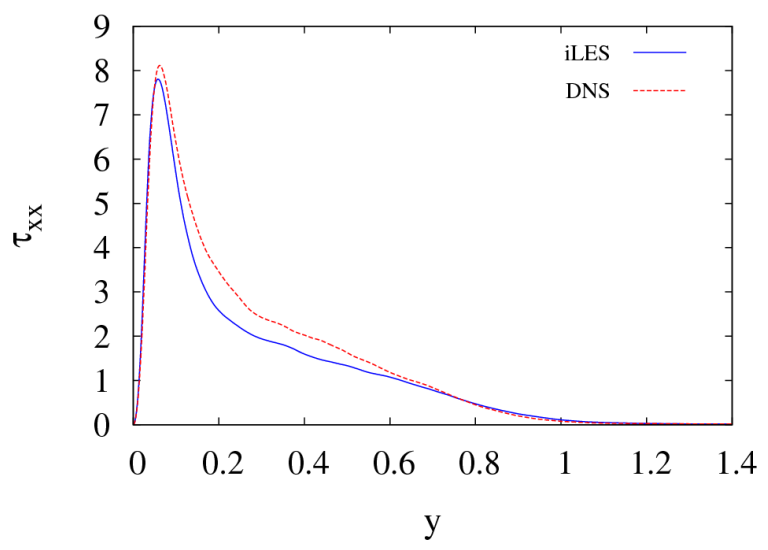

(a)

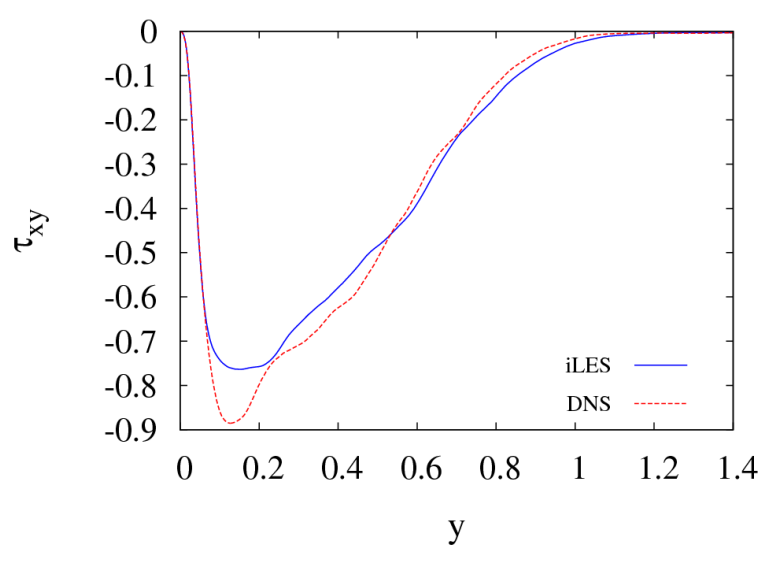

(c)

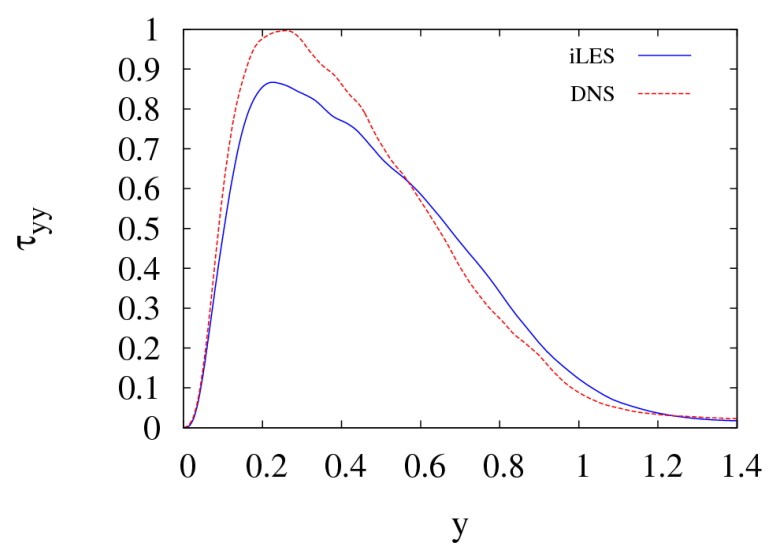

(b)

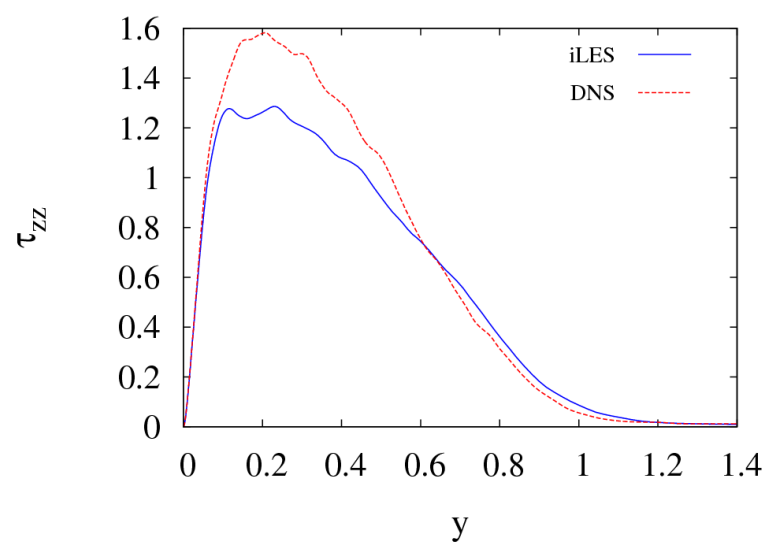

(d)

FIG. 6. Comparison of Reynolds stresses in inner coordinates at the end of the simulation domain for both iLES ${ }^{44}$ and DNS results: a) $\tau_{x x}$, b) $\tau_{y y}$, c) $\tau_{x y}$, d) $\tau_{z z}$

freestream flow from the flatness distribution, creating intermittent events above the boundary layer. Further high order statistics for all velocity components are available for both iLES and DNS cases, and detail analysis can give more insight into the flow physics and the effect of mesh resolution. It should also be noted that iLES plots of the same values (not shown here) would lead to the same observations and conclusions regarding the flow characteristics.

\section{B. Pressure fluctuations}

The results in the preceding section provide confidence in the accuracy of the simulations. The fundamental statistical quantity that describes the magnitude of near-wall acoustic loading is the root-mean-square level of the pressure fluctuation $\left(P_{r m s}^{\prime}\right)$. The standard approach is to non-dimensionalize it using the boundary layer edge or freestream dynamic pressure $q_{\infty}=\gamma P_{\infty} M^{2} / 2$. We present results in this form to be directly comparable to other numerical and experimental studies and other hypersonic cases such as the compression/expansion case. In an earlier publication ${ }^{19}$ we have compared our iLES results with an existing theoretical model, which predicts $P_{r m s}^{\prime} / q_{\infty}$ near the wall below fully turbulent boundary layers. In the same paper, we highlighted the accuracy of iLES by comparing with previous independent DNS studies at various Mach numbers, and we proposed a correction on the theoretical model to improve its accuracy on compressible supersonic and hypersonic flows.

Figure 8 shows that DNS predicts an earlier pressure fluctuation peak with an increased magnitude as compared with iLES. However, both iLES and DNS converge to the same pressure fluctuations in the fully turbulent region. This supports our findings on the correction of the theoretical model in ${ }^{19}$ and does not alter our previous conclusions. The above result demonstrates that iLES can predict pressure fluctuations and acoustic loading in fully turbulent areas without compromising accuracy compared to the intensive DNS.

In Figure 8, we have included the (approximate) iLES error associated with different numerical methods ranging from 5th to 9th order accuracy. Note that the estimated error has emerged from the authors' extensive experience us- 

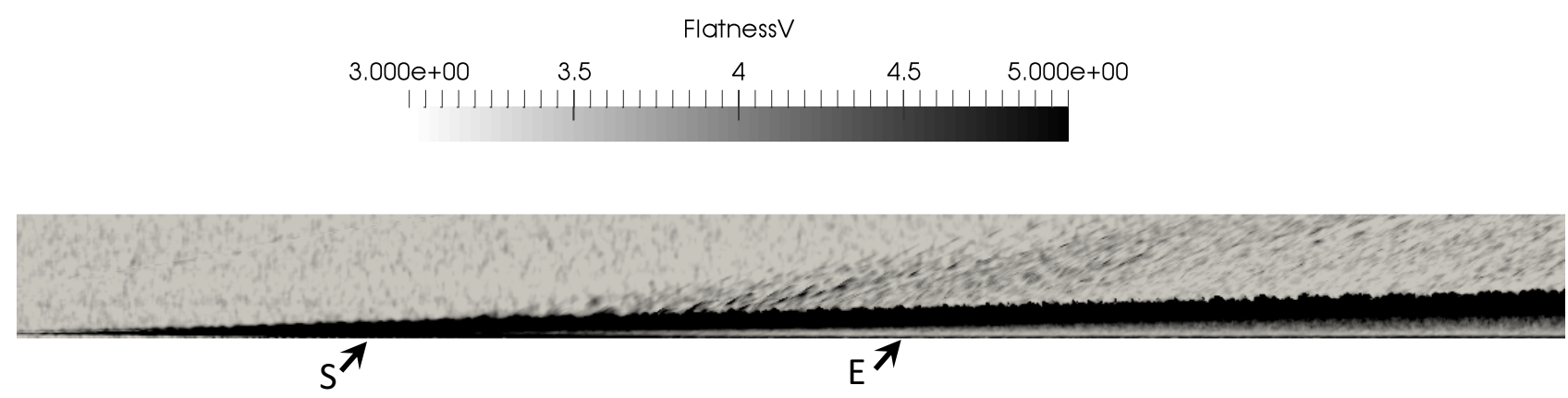

FIG. 7. Skewness and flatness of the streamwise $u^{\prime}$ and normal to the wall $v^{\prime}$ velocity fluctuations, respectively, with the latter one limited to a threshold value of 5 . Flatness is the fourth moment that shows the frequency of events far from the mean value. A value of flatness around 3 indicate a Gaussian or symmetric probability distribution function. High values of flatness indicate a highly intermittent flow. We also indicate the start- and end-of-the-transition points based on the minimum and maxim values of the skin friction, respectively.

ing high-order methods for iLES in various compressible flows ${ }^{30-32,39,57}$. The error ranges from $\pm 2 \%$ to $\pm 5 \%$ depending on the flow region, transitional or turbulent.

Pressure fluctuations have an essential effect on the structural response of hypersonic structures. The iLES delay in transition compared to DNS does not affect $\left(P_{r m s}^{\prime}\right)$ in the turbulent region. Since the results show that iLES is sufficiently accurate to predict pressure fluctuations in the turbulent regime, the delay in transition is not expected to affect the structural response. To corroborate the above, future aerostructure simulations that involve iLES over flexible panels are required.

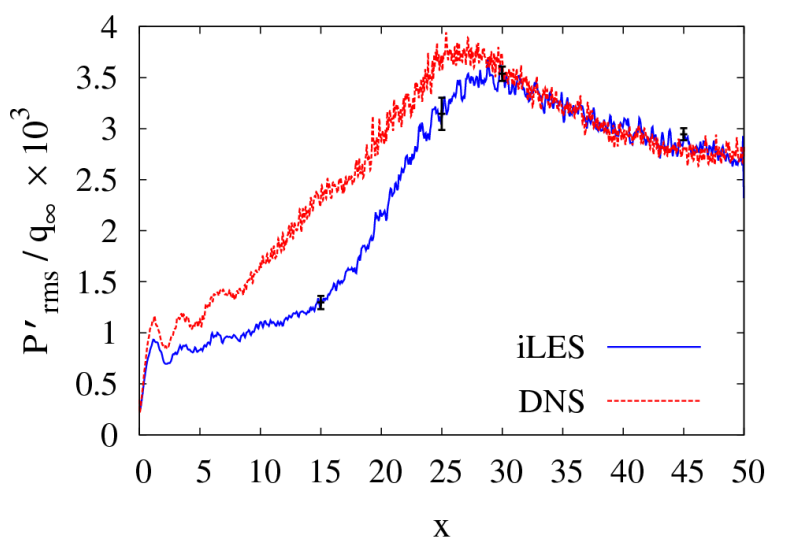

FIG. 8. Comparison of pressure fluctuations along the plate between iLES and DNS. The error bars (black color) on the iLES results correspond to $\pm 5 \%$ and $\pm 2 \%$ of the local value for the two selected points in the transition and turbulent region.
The DNS reveals the magnitude of the pressure fluctuations inside the boundary layer (Figure 9). The end of the transition region is visible by the high values of $P_{r m s}^{\prime}$ (red areas in the figure). Above the boundary layer edge, some pressure fluctuations appear in an angle linked to the freestream Mach number, contributing to far-field noise. These far-field fluctuations generated from the transition region can be correlated to velocity fluctuations in the same area, as observed in Figure 7.

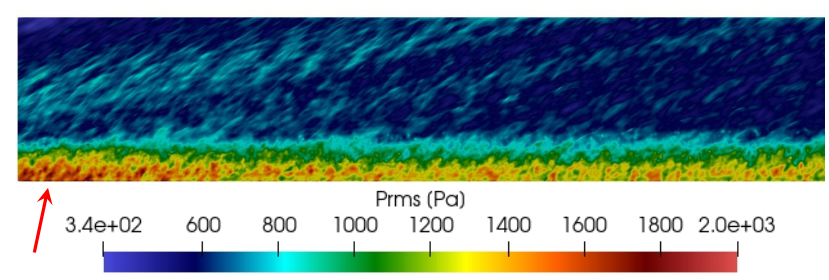

FIG. 9. Root mean square pressure fluctuations in the turbulent region highlighting the boundary layer thickness. The end-of-thetransition area can also be identified from the very high, near-wall values and approximately indicated with the red arrow.

In acoustic loading calculations, it is common to transform the fluctuating pressures to sound pressure level (SPL), which can be connected directly to high cycle fatigue in aerospace structures,

$$
S P L=20 \log _{10}\left(\frac{P_{r m s}^{\prime}}{20 \mu \mathrm{Pa}}\right) .
$$

The acoustic loads' peak in the transitional region, obtaining significantly higher values than those sustained in the laminar 


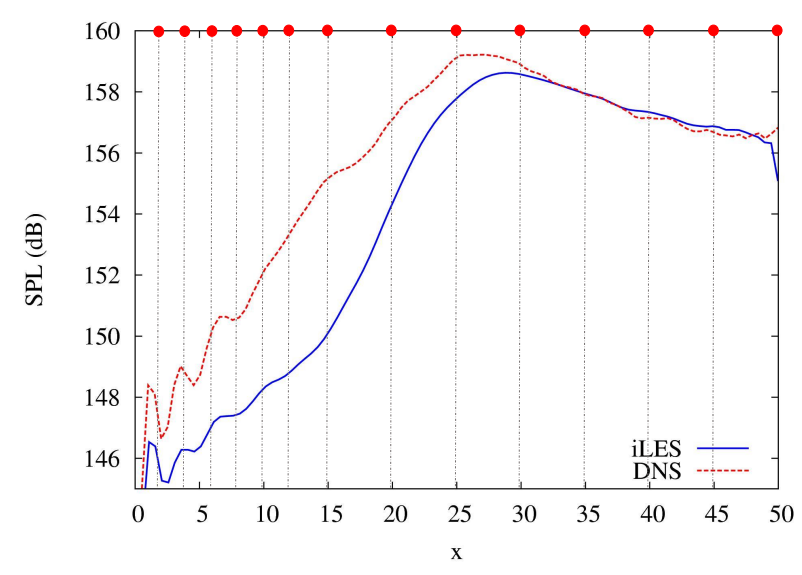

FIG. 10. Comparison of SPL along the plate between iLES and DNS, a Bezier function has been used for smoothing. On the upper axis of the figure we show the locations (red dots) of pressure history calculations.

or fully turbulent regions ${ }^{14,58}$. For both cases considered in this study, the pressure fluctuations in the transition region are $35 \%$ larger $(\sim 3 \mathrm{~dB})$ than their values in the fully turbulent area (Figure 10). The difference in acoustic loading between the laminar and transition region could be more than $10 \mathrm{~dB}$. In a previous study, the authors have shown that the inlet fluctuations' turbulent intensity influences the maximum acoustic load location, but not its value ${ }^{19}$. Downstream of the end of the transition, simulations with different turbulent intensities and $M=6$ converged to the same level of acoustic loading. Similarly, here we show that a high-order iLES in a relatively fine mesh can converge to the same acoustic loading level as a highly accurate and computationally expensive DNS.

We defined 42 specific places on the wall to monitor the pressure values at every timestep, leading to hundreds of thousands of samples with nanosecond resolution. Those locations are along with 14 points in the streamwise direction, indicated by a red dot in Figure 10, and 3 spanwise positions for every streamwise site. We chose the exact locations based on our previous iLES simulations, which lead to having samples in all three flow regimes (laminar, transitional and turbulent) and in critical areas like the position of peak acoustic loading $(x=25)$. Pressure time histories from DNS and iLES are shown in Figure 11. We have selected the points to be at the transition initiation, the end of the transition, and inside the fully turbulent regime. We also provide the mean value and standard deviation (STD) in Table IV. Regarding the mean and STD values, the agreement between iLES and DNS is remarkable in the turbulent region. More significant differences appear mainly in the transitional region, where due to the mesh resolution, DNS predicts larger fluctuation values. For $x=15$ the DNS's STD is almost twice that of iLES, but the difference reduces as we move towards the end of the transition region. In the following section, we perform spectral analysis of the pressure signal in those 4 locations for both iLES and DNS.
TABLE IV. Mean value and standard deviation (STD) for the pressure time histories at the various positions shown in Figure 11.

\begin{tabular}{ccccc}
\hline \hline$x$ & Mean $_{i L E S}$ & Mean $_{D N S}$ & STD $_{i L E S}$ & STD $_{D N S}$ \\
\hline 15 & 0.04004 & 0.04014 & 0.00125 & 0.00248 \\
\hline 25 & 0.04100 & 0.04122 & 0.00284 & 0.00389 \\
\hline 30 & 0.04240 & 0.04194 & 0.00354 & 0.00338 \\
\hline 45 & 0.04208 & 0.04193 & 0.00268 & 0.00268 \\
\hline
\end{tabular}

\section{Spectral Analysis of Acoustic Loading}

We analyze each pressure history signal as a single-point spectrum in the frequency domain, which is defined by

$$
\Phi(\omega)=\frac{1}{2 \pi} \int_{-\infty}^{\infty} \overline{P^{\prime}(x, y, z, t) P^{\prime}(x, y, z, t+\tau)} e^{-i \omega \tau} d \tau
$$

where $\tau$ is a time delay and $\omega$ is the radial frequency. Initially, we evaluate the iLES approach's accuracy in direct comparison with DNS results at the same locations (see Figure 12). Despite the significant lower resolution in both space and time, the iLES results have excellent agreement with the DNS in the low and medium frequency regimes. In higher frequencies, the iLES underestimates the spectrum's amplitude, while we cannot resolve very high frequencies due to the mesh size. The spectrum roll-off follows the same scalings in all cases, as will show in more detail below. It is worth mentioning that DNS can capture at over $21 \mathrm{MHz}$.At the same time, iLES can resolve frequencies up to $4.3 \mathrm{MHz}$, which is still significantly higher than most experimental sensors and measurement techniques can achieve $e^{20,21,60}$. The frequency range accurately captured by the simulations is limited by the mesh resolution and time. The maximum resolved frequency depends on mesh resolution, hence the fivefold difference between iLES and DNS. The minimum resolved frequency depends on time; longer simulation times resolve lower frequencies. In the present results, the minimum frequency captured is around $350 \mathrm{kHz}$.

Figure 12(a) shows the spectra at the peak of the transition for the DNS case $(x=25)$. Knowing from the analysis in the previous sections that iLES reaches the peak at a later point, we include at the same plot the DNS results from location $x=20$ where the magnitude of the pressure fluctuations is closer to that of iLES at $x=25$. In that case, the spectra are identical up to the maximum resolution that iLES can achieve. This is a possible indication that the mismatch at higher frequencies when we compare the spectra between iLES and DNS at the same locations could be potentially attributed to the iLES delay in reaching the transition's peak.

In the same set of figures, we also include a semi-empirical wall-pressure spectra model (COMPRA-G), which the authors have extended based on Goody's model ${ }^{61}$. More discussion about semi-empirical models and COMPRA-G can be found in ${ }^{62}$. The initially proposed model produces a scaling behaviour based on the established pressure spectrum scaling for zero pressure-gradient, fully turbulent subsonic and supersonic flows. The proposed COMPRA-G is also applicable to high-speed flows, as we include compressibility ef- 


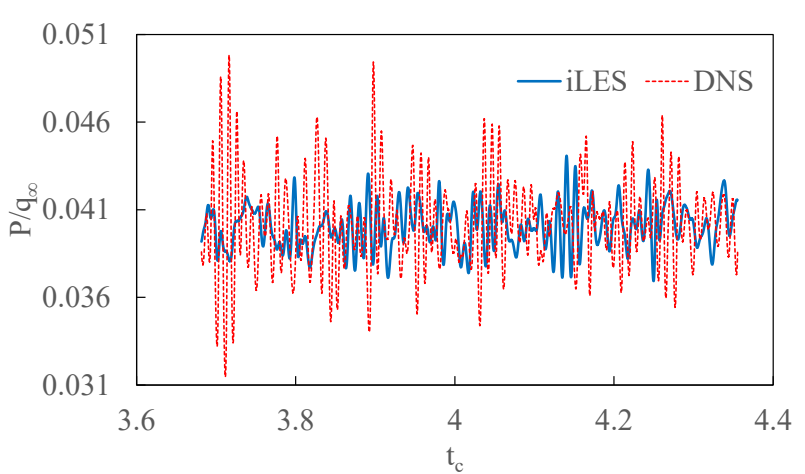

(a)

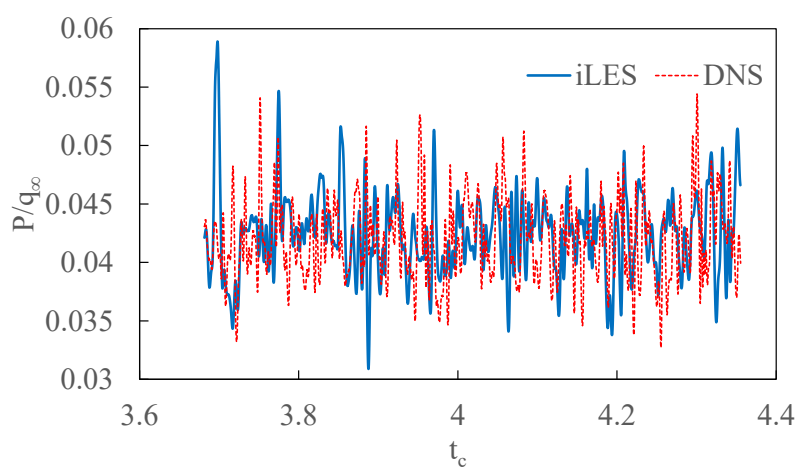

(c)

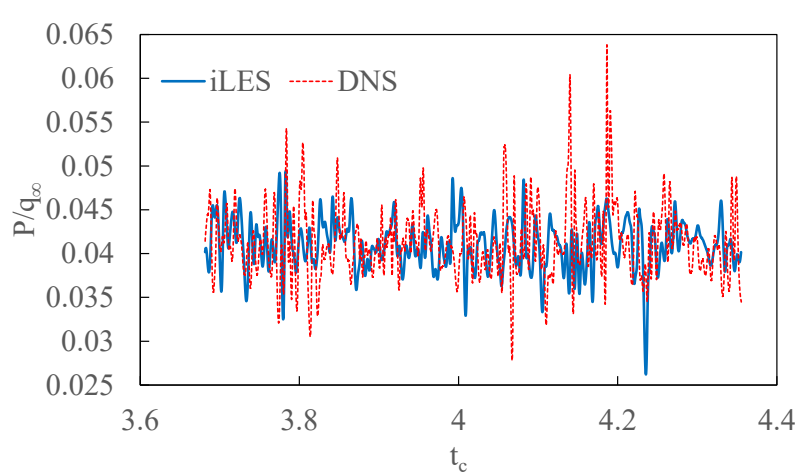

(b)

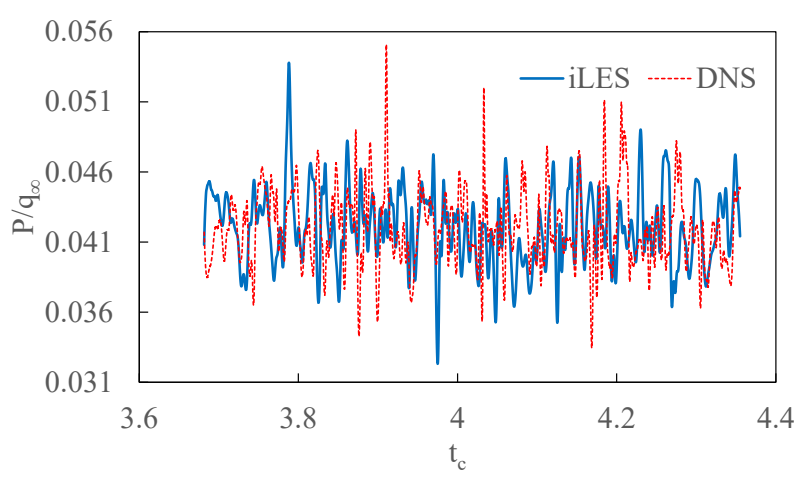

(d)

FIG. 11. Comparison of pressure time histories between iLES and DNS at the centerline. The time, $t_{c}$, has been non-dimensionalized by the time required for one flow cycle: a) $x=15$, b) $x=25$, c) $x=30$, and d) $x=45$.

fects. COMPRA-G also retains the excellent prediction capabilities at lower-speed flows down to the incompressible limit $(M=0.1)^{62}$.

COMPRA-G consists of one equation, based on Howe's ${ }^{63}$ and Goody's ${ }^{61}$ past studies:

$$
\frac{\Phi(\omega) u_{\infty}}{\tau_{w}^{2} \delta}=\frac{C_{2} \chi^{2}}{\left[\chi^{0.75}+C_{1}\right]^{3.7}+\left[C_{3} R_{T}^{C_{R G}} \chi\right]^{7}},
$$

where $C_{1}=0.5, C_{2}=3.0$ and $C_{3}=1.1$ are empirical constants, $\chi=\omega \delta / u_{\infty}, R_{T}=\delta u_{\tau}^{2} /\left(v_{w} u_{\infty}\right)$ and $C_{R G}=-0.49$ for supersonic and hypersonic flows. In the case of incompressible flows $C_{R G}=-0.57$. The authors have shown ${ }^{62}$ that all the necessary parameters for the calculation of COMPRA-G can be based on freestream properties, the distance from the leading edge and empirical correlations, like the one proposed by Bies $^{59}$ for the boundary layer thickness

$$
\delta=0.37 x \operatorname{Re}_{x}^{-0.2}\left[1+\left(\frac{R e_{x}}{6.9 \times 10^{7}}\right)^{2}\right]^{0.1}
$$

We show characteristic values of $\delta$ for each location in Figure 12 based on Eq. 12, where $x$ is the distance from the leading edge of the plate in meters. The spectra from the iLES and DNS calculations have been non-dimensionalized based on parameters calculated during the simulations at the corresponding locations.

The COMPRA-G empirical model can capture the low and medium frequency regimes with high accuracy based only on freestream properties usually known or at least much easier to obtain. High frequencies are not captured correctly as the model has been designed with an $\omega^{-5}$ scaling, while hypersonic flows seem to have steeper high-frequency scalings. COMPRA-G can predict the pressure spectra below fully turbulent boundary layers and in the challenging transitional regimes. Such a model could be used for quick approximations when the focus is at the low and medium frequency regimes without more expensive simulations or experiments.

The spectra results can also be analyzed in comparison with the theoretical predictions for fully turbulent flows ${ }^{4}$, and various observations ${ }^{64}$. Bull ${ }^{64}$ isolated four different regions of low, mid (which includes the spectral peak), mid-to-high overlap, and high frequencies, with corresponding spectrum slopes of $\omega^{2}, \omega^{0}, \omega^{-r}(r=[0.7,1.1])$, and $\omega^{-t}(t=[7 / 3,5])$, respectively. The turbulent motion influences the low-frequency region in the outer part of the boundary layer. The viscosity and turbulent motion affect high frequencies in the inner part of the boundary layer.

According to the theoretical arguments made by FfowcsWilliams $^{2}$ for compressible flows, in the low-frequency region, the scaling should be $\omega \rightarrow 0$. This observation has been 


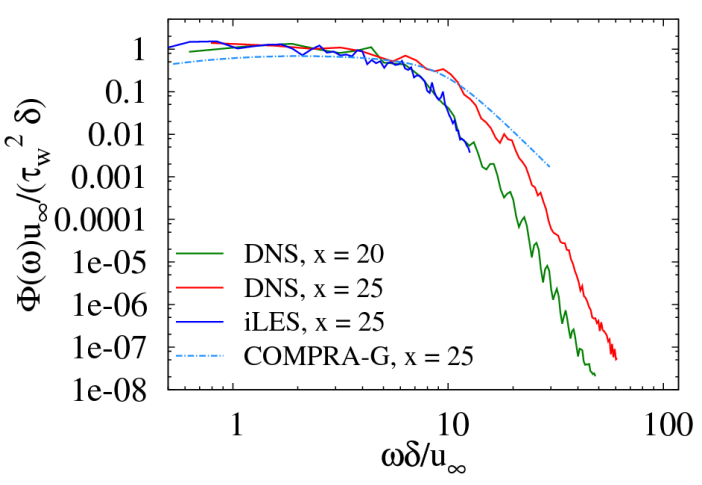

(a)

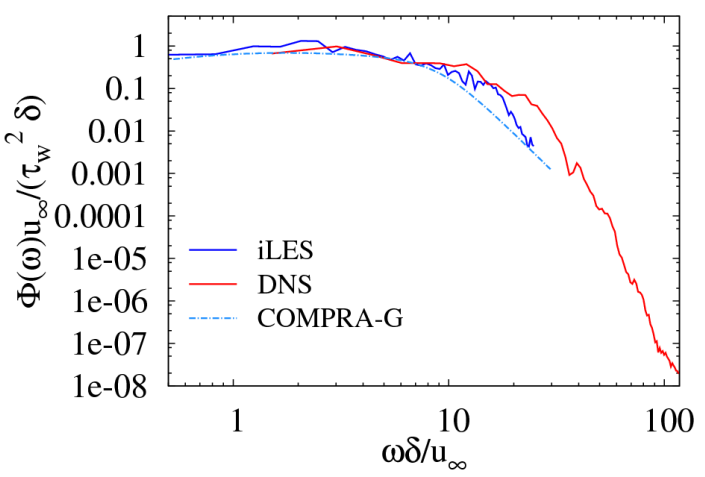

(b)

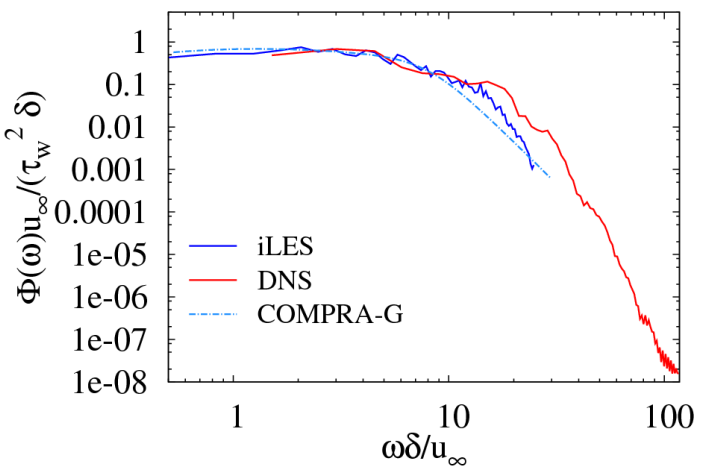

(c)

FIG. 12. Comparison of spectra between iLES, DNS and the COMPRA-G model at various locations in the transitional and fully turbulent regime. In a) we also present the spectrum of the pressure history at location $x=20$ from DNS data: a) $x=25$ and $\delta=1.05$ mm based on Bies ${ }^{59}$ empirical correlation for the calculation of $\delta ;$ b) $x=30, \delta=1.21 \mathrm{~mm}$; c) $x=45, \delta=1.66 \mathrm{~mm}$.

confirmed by experimental and numerical studies of supersonic and hypersonic turbulent boundary layers $8,9,18,19,65$. The above is in contrast to the Kraichnan-Phillips theorem for incompressible flows ${ }^{64,66,67}$, which suggests $\omega^{2}$.

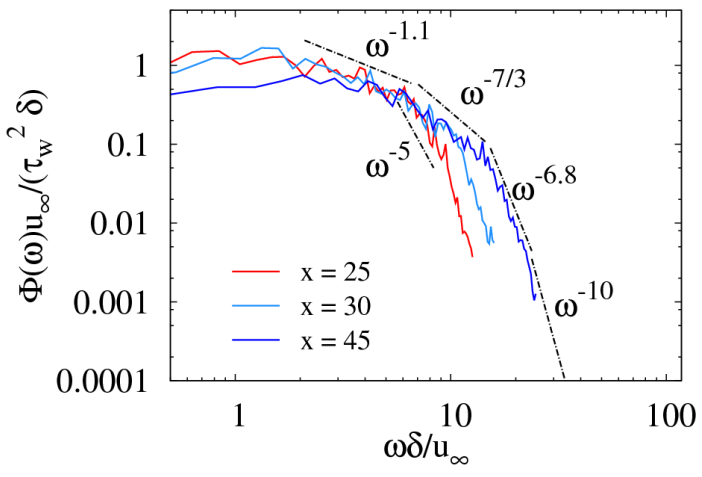

(a) iLES

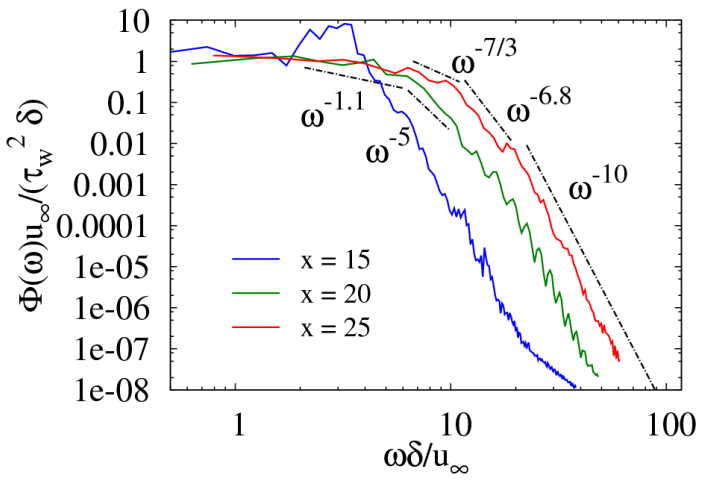

(b) Transition region - DNS

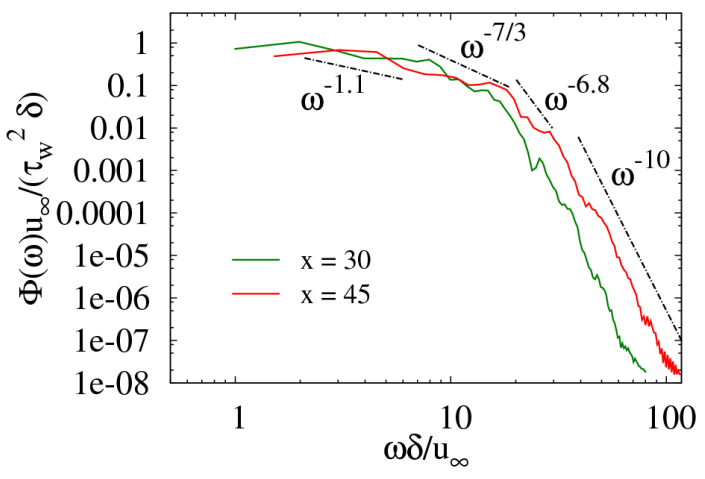

(c) Turbulent region - DNS

FIG. 13. PSD development from transitional to turbulent regimes produced by a) iLES and b-c) DNS data.

The mid-to-high overlap frequency region appears at sufficiently high $\operatorname{Re}_{\theta}$ values and the spectrum varies as $\omega^{-r}$ with $r=0.7$ to 1.1 , influenced by the local Reynolds number. This region is associated with pressure-induced eddies in the logarithmic region of the boundary layer. Its scaling behaviour was predicted by Bradshaw ${ }^{3}$ and was verified theoretically ${ }^{68}$ and experimentally ${ }^{6,8}$.

Following the $\omega^{-r}$ region, the spectrum becomes $\omega^{-7 / 3}$, 
henceforth called "acoustic-transition", which was also predicted for isotropic turbulence by Batchelor ${ }^{69}$ and has also been observed in various experiments ${ }^{70-72}$, as well as verified by numerical calculations of supersonic turbulent boundary layers $^{9,18}$. The spectrum decays more rapidly at high frequencies, reaching a slope proportional to $\omega^{-5}$. Sources in the sublayer $\left(y^{+}<20\right)$ contribute to this frequency region according to the theoretical prediction of Blake ${ }^{4}$, with the scaling being validated experimentally, as well ${ }^{5,6}$.

In Figure 13 we focus on the scaling of the spectrum at the various frequency regimes for both iLES (Figure 13(a)) and DNS results (Figures 13(b), 13(c)), including data obtained below both transitional and fully hypersonic turbulent boundary layers. In all cases, the low-frequency region yields $\omega \rightarrow 0$ and the mid-to-high overlap frequency region scales as $\omega^{-1.1}$, which both agree with the analysis above. The overlap region occurs over a broader range of frequencies compared to previous studies at lower Mach numbers, and observations also made previously by the authors for hypersonic flows ${ }^{19}$.

The high-frequency region of the spectrum, including the "acoustic-transition" scaling, also appears here in all turbulent boundary layers. However, the high-frequency region scales with $\omega^{-6.8}$. The above slope is steeper than the usually observed $\omega^{-5}$. On top of that, we can follow a region of $\omega^{-10}$ at very high frequencies. This last region is difficult to observe in the iLES results due to resolution restrictions. Still, it has been already mentioned, albeit with a smaller value, by Ritos et al. ${ }^{19}$ for flows in a range of Mach number and turbulent intensities. We attribute this region to high-speed, compressibility effects closer to the wall $\left(y^{+}<20\right)$. The Mach dependence of the spectrum in fully turbulent boundary layers has also been observed in experiments ${ }^{73}$, and numerical simulations ${ }^{18}$.

Figure 13(b) shows the spectrum roll-off development in the transitional regime through the DNS results. The spectra towards the end of this region resemble those observed in a fully turbulent flow. However, at $x=15$, close to the startof-the-transition point, the spectrum presents a previously unobserved PSD jump at the mid-frequency range. This point is far from the literature's conditions, and deviations from the existing theory regarding the spectrum roll-off below fully turbulent boundary layers are expected. We do not present iLES results for this point due to mesh restrictions which limit us to the (flat) low-frequency region.

From further analysis of Figure 13, it becomes apparent that the high-frequency region is different in transitional boundary layers and before the peak of the transition $(x=25$ for iLES and $x=20$ for DNS). The "acoustic-transition" scaling is not possible, and the high-frequency region presents a slope proportional to the usually observed $\omega^{-5}$ before becoming steeper and equal to $\omega^{-10}$ at the very high-frequency end. It is essential to mention here that iLES can resolve the correct scaling behaviour under both transitional and turbulent boundary layers despite the significantly reduced resolution compared to the DNS.

\section{CONCLUSIONS}

The present study results shed light on the accuracy of highresolution simulation approaches such as implicit Large Eddy Simulation and Direct Numerical Simulation for hypersonic transitional and turbulent flows and associated acoustic loading near-wall effects. The main conclusions drawn from this research are summarized below:

1. iLES can produce highly accurate results in both flow and acoustic metrics even though a 50 times reduced mesh resolution compared to DNS.

2. iLES slightly under-predicts the transition peak and delays the transition.

3. iLES predicts the correct spectrum roll-off for transitional and turbulent hypersonic boundary layers. However, it underpredicts high frequencies' amplitude due to mesh resolution constraints at high frequencies.

4. The acoustic spectra scaling at Mach 6 is different than that of incompressible flow across frequencies. Therefore, incompressible models should not be used in highspeed flows.

5. The proposed wall-pressure spectra model COMPRA$G$ that includes compressibility effects gives good estimates of the low and medium frequency regimes based only on freestream properties. This makes its implementation extremely straightforward and fast, avoiding the need for costly computer simulations.

6. Since the iLES captures the near-wall acoustic spectrum roll-off at low/medium frequencies, that would make it appropriate for modeling structural dynamic loading.

Regarding future work, we aim to use wind tunnel data in conjunction with high-resolution simulations to investigate pressure fluctuation near the wall further and advance the development of reduced-order models. The U.S. Air Force Research Laboratory (AFRL) Structural Sciences Center (SSC) leads an experimental campaign in the Arnold Engineering Development Center (AEDC) von Kármán Facility Tunnel C (VKF-C) to investigate the aerothermoelastic behavior of a flight-weight thin titanium panel at Mach 4, as well as other flow conditions in the future. Although the recent experiment at Mach 4 is at different freestream conditions and measurement positions in the boundary layer with those of the present paper, we found that the experiment's PSD at the lowfrequency $\left(0.3\right.$ to $\left.3 \omega \delta / u_{\infty}\right)$ resembles Mach 6 simulations with an $\omega^{0}$ scaling. The above is a promising result that justifies further investigation subject to the availability of new experiments that measure pressure fluctuations nearer the wall.

\section{ACKNOWLEDGMENTS}

This work was sponsored by the Air Force Office of Scientific Research, Air Force Material Command, USAF, under grant number FA9550-19-1-7018. The U.S. Government 
is authorised to reproduce and distribute reprints for Governmental purpose notwithstanding any copyright notation thereon. The authors would like to thank Dr Garner for his support.

\section{DATA AVAILABILITY}

The data that support the findings of this study are available on request from the authors. The data are not publicly available due to the funding body restrictions.

${ }^{1}$ A. L. Laganelli, A. Martellucci, and L. L. Shaw, "Wall pressure fluctuations in attached boundary-layer flow," AIAA Journal 21, 495-502 (1983).

${ }^{2}$ J. E. Ffowcs-Williams, "Surface pressure fluctuations induced by boundary layer flow at finite Mach number," J. Fluid Mech. 22, 507-519 (1965).

${ }^{3} \mathrm{P}$. Bradshaw, "Inactive motion and pressure fluctuations in turbulent boundary layers," J. Fluid Mech. 30, 241-258 (1967).

${ }^{4}$ W. K. Blake, Mechanics of Flow-Induced Sound and Vibration (New York: Academic Press, 1986).

${ }^{5}$ T. M. Farabee and M. J. Casarella, "Spectral features of wall pressure fluctuations beneath turbulent boundary layers," Phys. Fluids A 3, 2410-2420 (1991).

${ }^{6}$ S. P. Gravante, A. M. Naguip, C. E. Wark, and H. M. Nagib, "Characterization of the pressure fluctuations under a fully developed turbulent boundary layer," AIAA Journal 36, 1808-1816 (1998).

${ }^{7}$ Y. Tsuji, J. H. N. Fransson, P. H. Alfredsson, and A. V. Johansson, "Pressure statistics and their scaling in high-reynolds-number turbulent boundary layers," J. Fluid Mech. 585, 1-40 (2007).

${ }^{8}$ S. J. Beresh, J. F. Henfling, R. W. Spillers, and B. O. M. Pruett, "Fluctuating wall pressures measured beneath a supersonic turbulent boundary layer," Phys. Fluids 23, 1-16 (2011).

${ }^{9}$ M. Bernardini, S. Pirozzoli, and F. Grasso, "The wall pressure signature of transonic shock/boundary layer interaction," Journal of Fluid Mechanics 671, 288-312 (2011).

${ }^{10} \mathrm{~J}$. Poggie, N. J. Bisek, and R. Gosse, "Resolution effects in compressible, turbulent boundary layer simulations," Computers \& Fluids 120, 5769 (2015).

${ }^{11}$ C.-H. Zhang, Q. Tang, and C.-B. Lee, "Hypersonic boundary-layer transition on a flared cone," Acta Mech. Sin. 29, 48-53 (2013).

${ }^{12}$ K. M. Casper, S. J. Beresh, and S. P. Schneider, "Pressure fluctuations beneath instability wavepackets and turbulent spots in a hypersonic boundary layer," J. Fluid Mech. 756, 1058-1091 (2014).

${ }^{13}$ Y. Zhu, C. Zhang, X. Chen, H. Yuan, J. Wu, S. Chen, and C. Lee, "Transition in hypersonic boundary layers: Role of dilatational waves," AIAA Journal 54, 3039-3049 (2016).

${ }^{14}$ K. M. Casper, S. J. Beresh, J. F. Henfling, R. W. Spillers, and B. O. M. Pruett, "Hypersonic wind-tunnel measurements of boundary-layer transition on a slender cone," AIAA Journal 54, 1250-1263 (2016).

${ }^{15}$ L. Duan, I. Beekman, and M. P. Martín, "Direct numerical simulation of hypersonic turbulent boundary layers. part 3. effect of mach number," J. Fluid Mech. 672, 245-267 (2011).

${ }^{16}$ K. J. Franko and S. K. Lele, "Breakdown mechanisms and heat transfer overshoot in hypersonic zero pressure gradient boundary layers," J. Fluid Mech. 730, 491-532 (2013).

${ }^{17}$ J. Sivasubramanian and H. F. Fasel, "Direct numerical simulation of transition in a sharp cone boundary layer at mach 6: fundamental breakdown," J. Fluid Mech. 768, 175-218 (2015).

${ }^{18}$ L. Duan, M. M. Choudhari, and C. Zhang, "Pressure fluctuations induced by a hypersonic turbulent boundary layer," Journal of Fluid Mechanics 804, 578-607 (2016).

${ }^{19}$ K. Ritos, D. Drikakis, and I. Kokkinakis, "Acoustic loading beneath hypersonic transitional and turbulent boundary layers," Journal of Sound and Vibration 441, 50 - 62 (2019).

${ }^{20} \mathrm{X}$. Zhao and L. Zhao, "Wall pressure fluctuations beneath hypersonic boundary layer over plate," AIAA Journal , 1-10 (2020).
${ }^{21}$ J. Huang, L. Duan, K. Casper, R. Wagnild, and N. Bitter, "Direct numerical simulation of turbulent pressure fluctuations over a cone at Mach 8," in AIAA SicTech Forum, AIAA 2020-1065 (2020).

${ }^{22}$ L. M. Mack, "Boundary layer linear stability theory," Tech. Rep. 709 (AGARD, 1984).

${ }^{23}$ R. E. Spall and M. R. Malik, "Goertler vortices in supersonic and hypersonic boundary layers," Physics of Fluids A: Fluid Dynamics 1, 1822-1835 (1989).

${ }^{24}$ N. M. El-Hady, "Nonparallel instability of supersonic and hypersonic boundary layers," Physics of Fluids A: Fluid Dynamics 3, 2164-2178 (1991).

${ }^{25}$ M. Lagha, J. Kim, J. D. Eldredge, and X. Zhong, "A numerical study of compressible turbulent boundary layers," Physics of Fluids 23, 015106 (2011).

${ }^{26}$ Y. Zhu, X. Chen, J. Wu, S. Chen, C. Lee, and M. Gad-el Hak, "Aerodynamic heating in transitional hypersonic boundary layers: Role of secondmode instability," Physics of Fluids 30, 011701 (2018).

${ }^{27}$ J. Liu, J. Xu, C. Wang, P. Yu, and J. Bai, "Pressure gradient effects on the secondary instability of mack mode disturbances in hypersonic boundary layers," Physics of Fluids 33, 014109 (2021).

${ }^{28}$ L. Mack, "Linear stability theory and the problem of supersonic boundarylayer transition," AIAA Journal 13, 278-285 (2012).

${ }^{29}$ I. Kokkinakis, D. Drikakis, K. Ritos, and S. M. Spottswood, "Direct numerical simulation of supersonic flow and acoustics over a compression ramp," Physics of Fluids 32, 066107 (2020).

${ }^{30}$ D. Drikakis, M. Hahn, A. Mosedale, and B. Thornber, "Large eddy simulation using high resolution and high order methods," Proc. R. Soc. A 367, 2985-2997 (2009).

${ }^{31}$ K. Ritos, I. W. Kokkinakis, D. Drikakis, and S. M. Spottswood, "Implicit large eddy simulation of acoustic loading in supersonic turbulent boundary layers," Physics of Fluids 29, 046101 (2017).

${ }^{32}$ K. Ritos, I. W. Kokkinakis, and D. Drikakis, "Physical insight into the accuracy of finely-resolved iLES in turbulent boundary layers," Computers \& Fluids 169, 309-316 (2018).

${ }^{33}$ F. F. Grinstein, L. G. Margolin, and W. J. Rider, "Implicit large eddy simulation: computing turbulent fluid dynamics," Cambridge University Press (2007).

${ }^{34}$ D. Drikakis and B. Geurtsr, "Turbulent flow computation," Springer (2002).

${ }^{35}$ E. F. Toro, M. Spruce, and W. Speares, "Restoration of the contact surface in the HLL-Riemann solver," Shock Waves 4, 25 - 34 (1994).

${ }^{36}$ E. F. Toro, Riemann Solvers and Numerical Methods for Fluid Dynamics, 3rd ed. (Springer, 2009).

${ }^{37}$ A. K. Henrick, T. D. Aslam, and J. M. Powers, "Mapped weighted essentially non-oscillatory schemes: Achieving optimal order near critical points," Journal of Computational Physics 207, 542 - 567 (2005).

${ }^{38}$ R. Spiteri and S. Ruuth, "A new class of optimal high-order strong-stabilitypreserving time discretization methods," SIAM Journal on Numerical Analysis 40, 469-491 (2002).

${ }^{39}$ I. Kokkinakis and D. Drikakis, "Implicit large eddy simulation of weaklycompressible turbulent channel flow," Computer Methods in Applied Mechanics and Engineering 287, 229 - 261 (2015).

${ }^{40}$ P. Tsoutsanis, I. W. Kokkinakis, L. Könözsy, D. Drikakis, R. J. Williams, and D. L. Youngs, "Comparison of structured- and unstructured-grid, compressible and incompressible methods using the vortex pairing problem," Computer Methods in Applied Mechanics and Engineering 293, 207 - 231 (2015).

${ }^{41}$ D. Drikakis, C. Fureby, F. Grinstein, and D. Youngs, "Simulation of transition and turbulence decay in the Taylor-Green vortex," Journal of Turbulence 8, 1-12 (2007).

${ }^{42}$ L. G. Margolin, W. J. Rider, and F. F. Grinstein, "Modeling turbulent flow with implicit les," J. Turbul. 7 (2006).

${ }^{43}$ C. Fureby and F. F. Grinstein, "Large eddy simulation of high-Reynoldsnumber free and wall-bounded flows," Journal of Computational Physics 181, 68 - 97 (2002)

${ }^{44}$ K. Ritos, D. Drikakis, , I. W. Kokkinakis, and S. M. Spottswood, "Computational aeroacoustics beneath high speed transitional and turbulent boundary layers," Computers and Fluids 203, 104520 (2020).

${ }^{45} \mathrm{X}$. Wu, J. M. Wallace, and J.-P. Hickey, "Boundary layer turbulence and freestream turbulence interface, turbulent spot and freestream turbulence 
interface, laminar boundary layer and freestream turbulence interface," Physics of Fluids, 045104 (2019).

${ }^{46}$ J. Blazek, Fluid Dynamics, Principles and Applications (Elsevier, 2015).

${ }^{47}$ P. Costamagna, G. Vittori, and P. Blondeux, "Coherent structures in oscillatory boundary layers," J. Fluid Mech. 474, 1-33 (2003).

${ }^{48}$ L. Duan and M. M. Choudhari, "Numerical study of pressure fluctuations due to a mach 6 turbulent boundary layer," in 51st AIAA Aerospace Sciences Meeting (2013) pp. 1-16.

${ }^{49}$ S. Stolz and N. A. Adams, "Large-eddy simulation of high-reynoldsnumber supersonic boundary layers using the approximate deconvolution model and a rescaling and recycling technique," Phys. Fluids 15 (2003).

${ }^{50} \mathrm{~S}$. Stolz, "High-pass filtered eddy-viscosity models for large-eddy simulations of compressible wall-bounded flows," J. Fluids Eng 127, 666-673 (2005).

${ }^{51}$ I. Marusic, B. J. McKeon, P. A. Monkewitz, H. M. Nagib, A. J. Smits, and K. R. Sreenivasan, "Wall-bounded turbulent flows at high reynolds numbers: Recent advances and key issue," Phys. Fluids 22 (2010).

${ }^{52}$ T. Gatski and J.-P. Bonnet, Compressibility, Turbulence and High Speed Flow (Academic Press, 2013).

${ }^{53}$ L. P. Erm, Low Reynolds-number turbulent boundary layers, Ph.D. thesis, Univ. of Melbourne (1988).

${ }^{54}$ J. Kim, P. Moin, and R. Moser, "Turbulence statistics in fully developed channel flow at low reynolds number," J. Fluid Mech. 177, 133-166 (1987).

${ }^{55}$ J. Jimenez and A. Pinelli, "The autonomous cycle of near-wall turbulence," J. Fluid Mech. 389, 335-359 (1999).

${ }^{56}$ S. Pirozzoli and M. Bernardini, "Turbulence in supersonic boundary layers at moderate Reynolds number," J. Fluid Mech. 688, 120-168 (2011).

${ }^{57}$ P. Tsoutsanis, I. W. Kokkinakis, L. Konozsy, D. Drikakis, R. J. R. Williams, and D. L. Youngs, "Comparison of structured and unstructured-grid, compressible and incompressible methods using the vortex pairing problem," Comput. Method. Appl. M. 293, 207-231 (2015).

${ }^{58}$ A. Martellucci, L. Chaump, D. Rogers, and D. Smith, "Experimental determination of the aeroacoustic environment about a slender cone," AIAA Journal 11, 635-642 (1973)

${ }^{59} \mathrm{D}$. A. Bies, "A review of flight and wind tunnel measurements of boundary layer pressure fluctuations and induced structural response," Tech. Rep.
NASA-CR-626 (NASA, 1965)

${ }^{60}$ S. M. Spottswood, T. J. Beberniss, T. G. Eason, R. A. Perez, J. M. Donbar, D. A. Ehrhardt, and Z. B. Riley, "Exploring the response of a thin, flexible panel to shock-turbulent boundary-layer interactions," Journal of Sound and Vibration 443, 74 - 89 (2019).

${ }^{61}$ M. Goody, "Empirical spectral model of surface pressure fluctuations," AIAA Journal 42, 1788-1794 (2004).

${ }^{62}$ K. Ritos, D. Drikakis, and I. W. Kokkinakis, "Wall-pressure spectra models for supersonic and hypersonic turbulent boundary layers," Journal of Sound and Vibration 443, 90 - 108 (2019).

${ }^{63}$ M. S. Howe, Acoustics of Fluid-structure Interactions (Cambridge University Press, 1998).

${ }^{64} \mathrm{M}$. Bull, "Wall-pressure fluctuations beneath turbulent boundary layers: some reflections on forty years of research," Journal of Sound and Vibration 190, 299 - 315 (1996).

${ }^{65}$ C. Zhang, L. Duan, and M. M. Choudhari, "Effect of wall cooling on boundary-layer-induced pressure fluctuations at mach 6," J. Fluid Mech. 822, 5-30 (2017).

${ }^{66}$ R. H. Kraichnan, "Pressure fluctuations in turbulent flow over a flat plate," J. Acoust. Soc. Am. 28, 378-390 (1956).

${ }^{67} \mathrm{O}$. M. Phillips, "On the aerodynamic surface sound from a plane turbulent boundary," Proc. R. Soc. A 234, 327-335 (1956).

${ }^{68}$ R. L. Panton and J. H. Linebarger, "Wall pressure spectra calculations for equilibrium boundary layers," J. Fluid Mech. 65, 261-287 (1974).

${ }^{69}$ G. K. Batchelor, "Pressure fluctuations in isotropic turbulence," Proc. Camb. Phil. Soc. 47, 359-374 (1951).

${ }^{70} \mathrm{G}$. Schewe, "On the Structure and Resolution of Wall-Pressure Fluctuations Associated with Turbulent Boundary Layer Flow,” J. Fluid Mech. 134, 311-328 (1983).

${ }^{71}$ Y. Tsuji and T. Ishihara, "Similarity scaling of pressure fluctuation in turbulence,” Phys. Rev. E 68, 026309 (2003).

${ }^{72}$ R. Camussi, M. felli, F. Pereira, G. Aloisio, and A. D. Marco, "Statistical properties of wall pressure fluctuations over a forward-facing step," Phys. Fluids 20, 075113 (2008).

${ }^{73}$ J. Laufer, "Some statistical properties of the pressure field radiated by a turbulent boundary layer," Phys. Fluids 7, 1191-1197 (1964). 